\title{
THE OSCULATING SOLID OF A CERTAIN CURVE IN [4]
}

\author{
by W. L. EDGE \\ (Received 1st July 1970)
}

1. The equation of the osculating plane at a point on the complete irreducible curve of intersection of two algebraic surfaces in [3] was found by Hesse (5, p. 283); the plane, having to contain the tangent of the curve, belongs to the pencil spanned by the tangent planes of the two surfaces, and it is a question of determining which plane of the pencil to choose. The equation also appears in the books of Salmon (6, p. 378) and Baker (1, p. 206). The analogous problem for the osculating solid at a point on the complete irreducible curve of intersection of three algebraic primals, or threefolds, in [4] does not appear to have been considered. The simplest instance is the octavic curve $C$ of intersection of three quadrics, and this has the special interest of being a canonical curve; moreover the quadrics are of the same order, and so can be replaced by any three linearly independent members of the net which they determine, a replacement of which it may be prudent to take advantage with a view to simplifying the algebra. It is a question of determining which solid to choose among the tangent solids to the quadrics of the net at a point on $C$, but while Hesse's methods serve to carry one a certain distance there seems no obvious way of pushing them to a conclusion. It is then natural, with a view to reaching a conclusion, to choose a net of quadrics that, through having some particular property, is more amenable. So let it be supposed that all the quadrics through $C$ have a common self-polar simplex $S$. If $S$ is the simplex of reference for homogeneous coordinates the quadrics can be taken $(4, \mathrm{p} .261)$ as

$$
\Sigma x_{j}^{2}=0, \quad \Sigma a_{j} x_{j}^{2}=0, \quad \Sigma a_{j}^{2} x_{j}^{2}=0,
$$

where summations, here and hereafter, run over $j=0,1,2,3,4$. Also, to prevent further specialization, no two of the five numbers $a_{j}$ are equal.

2. If $\xi$ is a point on $C$ the equation of the osculating solid, which has to contain the tangent of $C$, is, for appropriate $\lambda, \mu, v$,

$$
\Sigma\left(\lambda+\mu a_{j}+v a_{j}^{2}\right) \xi_{j} x_{j}=0 .
$$

Both $\xi$ and $d \xi$ are on the tangent, but the osculating solid must contain $d^{2} \xi$ and $d^{3} \xi$ as well, so that

$$
\Sigma\left(\lambda+\mu a_{j}+v a_{j}^{2}\right) \xi_{j} d^{2} \xi_{j}=\Sigma\left(\lambda+\mu a_{j}+v a_{j}^{2}\right) \xi_{j} d^{3} \xi_{j}=0 .
$$


But, by differentiating equations 1.1,

$$
\Sigma a_{j}^{k} \xi_{j} d \xi_{j}=\Sigma a_{j}^{k}\left(\xi_{j} d^{2} \xi_{j}+d \xi_{j}^{2}\right)=\Sigma a_{j}^{k}\left(\xi_{j} d^{3} \xi_{j}+3 d \xi_{j} d^{2} \xi_{j}\right)=0
$$

for $k=0,1,2$, so that the previous pair of equations is equivalent to

$$
\Sigma\left(\lambda+\mu a_{j}+v a_{j}^{2}\right) d \xi_{j}^{2}=\Sigma\left(\lambda+\mu a_{j}+v a_{j}^{2}\right) d \xi_{j} d^{2} \xi_{j}=0
$$

and the osculating solid of $C$ at $\xi$ is

$$
\left|\begin{array}{ccc}
\Sigma \xi_{j} x_{j} & \Sigma a_{j} \xi_{j} x_{j} & \Sigma a_{j}^{2} \xi_{j} x_{j} \\
\Sigma d \xi_{j}^{2} & \Sigma a_{j} d \xi_{j}^{2} & \Sigma a_{j}^{2} d \xi_{j}^{2} \\
\Sigma d \xi_{j} d^{2} \xi_{j} & \Sigma a_{j} d \xi_{j} d^{2} \xi_{j} & \Sigma a_{j}^{2} d \xi_{j} d^{2} \xi_{j}
\end{array}\right|=0 .
$$

One has now so to order matters that no differentials appear. To this end write

$$
f(\theta) \equiv\left(\theta-a_{0}\right)\left(\theta-a_{1}\right)\left(\theta-a_{2}\right)\left(\theta-a_{3}\right)\left(\theta-a_{4}\right) .
$$

Then, if $s_{k}=\Sigma a_{j}^{k} / f^{\prime}\left(a_{j}\right)$,

$$
s_{0}=s_{1}=s_{2}=s_{3}=0, s_{4}=1, s_{5}=\Sigma a_{j}=\sigma, \text { say. }
$$

The equations 1.1 for the five " unknowns" $x_{j}^{2}$ have a matrix of rank 3 , further specialization having been forestalled; their solutions are linearly dependent on two of them so that, if they are all satisfied when $x$ is $\xi$,

with $p, q$ arbitrary. Then

$$
\xi_{j}^{2} f^{\prime}\left(a_{j}\right)=p+q a_{j}
$$

so that

$$
\begin{aligned}
2 f^{\prime}\left(a_{j}\right) \xi_{j} d \xi_{j} & =d p+a_{j} d q, \\
4 f^{\prime}\left(a_{j}\right) d \xi_{j}^{2} & =\left(d p+a_{j} d q\right)^{2} /\left(p+a_{j} q\right),
\end{aligned}
$$

$$
\Sigma\left(p+a_{j} q\right)^{2} d \xi_{j}^{2}=0 \text { by } 2.1 \text {. }
$$

A second differentiation yields

$$
8 f^{\prime}\left(a_{j}\right) d \xi_{j} d^{2} \xi_{j}=\frac{2\left(d p+a_{j} d q\right)\left(d^{2} p+a_{j} d^{2} q\right)}{p+a_{j} q}-\frac{\left(d p+a_{j} d q\right)^{3}}{\left(p+a_{j} q\right)^{2}}
$$

so that, again by $2.1, \Sigma\left(p+a_{j} q\right)^{2} d \xi_{j} d^{2} \xi_{j}=0$. If, therefore, the columns of the determinant are multiplied by $p^{2}, 2 p q, q^{2}$ and then added zeros appear in the two bottom rows: the equation of the osculating solid is $\Sigma\left(p+a_{j} q\right)^{2} \xi_{j} x_{j}=0$. The differentials have vanished; it only remains for $p$ and $q$ to follow them. And this they immediately do since, by 2.1 and 2.2,

$$
\Sigma a_{j}^{3} \xi_{j}^{2}=q, \quad \Sigma a_{j}^{4} \xi_{j}^{2}=p+q \sigma
$$

the final form for the equation is

$$
\sum_{k=0}^{4}\left\{\Sigma a_{j}^{4} \xi_{j}^{2}-\left(\sigma-a_{k}\right) \Sigma a_{j}^{3} \xi_{j}^{2}\right\}^{2} \xi_{k} x_{k}=0
$$




\section{THE OSCULATING SOLID OF A CERTAIN CURVE IN [4]}

3. The equation has, up to this moment, been looked upon as controlling the freedom of $x ; x$ varies in a solid determined by the position of $\xi$ on $C$. But it can be regarded, in the opposite sense, as a restriction on $\xi$ with $x$ fixed; it is then the equation of a quintic primal whose 40 intersections $\xi$ with $C$ are the contacts with those of its osculating solids that pass through the given point $x$ in [4].

Unless one is cognisant of the matters expounded on pages 199 and 200 of (1) the number 40 is contrary to untutored expectation; one would, over hastily as it turns out, expect the number of osculating solids of $C$ through an arbitrary point $P$ to be not 40 but 80 , the number of sets $(7$, p. $188 ; 2$, p. 10 ; 8, p. 389) of a linear series $g_{8}^{3}$ that have four coincident members-the $g_{8}^{3}$ being that cut on $C$ by all solids through $P$. The resolution of this apparent contradiction must lie in the possession by $C$ of some peculiarity, a peculiarity which will be among those imported in specializing $C$ so that all the quadrics through $C$ have $S$ for a self-polar simplex. It is known (3, pp. 486-7) that the 40 intersections of this special curve $C$ with the bounding solids of $S$-eight with each of the five solids-are stalls: places where the osculating plane has 4-point, instead of the statutory minimal 3-point, intersection; a general canonical curve of genus 5 has no stalls. But when an osculating plane $\pi$ has 4-point intersection so has the solid joining $\pi$ to an arbitrary point $P$, so that the stall counts for four among the eight intersections of this solid with $C$, and is among the places where sets of $g_{8}^{3}$ have quadruple members. It is the $\mathbf{4 0}$ such places that are not stalls which furnish osculating solids through $P$.

4. The solution at the conclusion of $\$ 2$ of the problem here propounded leaves outstanding that of three quadrics whose common curve is not specialized; still more does it that of three primals, of orders $l, m, n$, whose common curve of order $\operatorname{lm} n$ is irreducible. The equation of the osculating solid at a point on this last curve will contain its coordinates to degree $10(l+m+n-5)$, and it remains to find it.

\section{REFERENCES}

(1) H. F. BAKer, Principles of Geometry Vol. 5 (Cambridge 1933).

(2) H. F. BAKer, Principles of Geometry Vol. 6 (Cambridge 1933).

(3) W. L. Edge, Humbert's plane sextics of genus 5, Proc. Cambridge Philos. Soc. 47 (1951), 483-495.

(4) W. L. Edge, The tacnodal form of Humbert's sextic, Proc. Royal Soc. Edinburgh Sect. A 68 (1970), 257-269.

(5) O. Hesse, Ưber die Wendepunkte der algebraischen ebenen Kurven und die Schmiegungs-Ebenen der Kurven von doppelter Krummung, welche durch den Schnitt zweier algebraischen Oberflächen entstehen, Journal für die reine und angewandte Mathematik 41 (1851), 272-284; Gesammelte Werke (Munich, 1897), 263-278. 
(6) G. SALmon, A treatise on the analytic geometry of three dimensions Vol. 1 (Dublin 1914).

(7) F. SEVERI, Vorlesungen über algebraische Geometrie (Leipzig 1921).

(8) J. G. SEMPLE and L. ROTH, Introduction to algebraic geometry (Oxford 1949).

Mathematical Institute

20 ChAMbers STREeT

EDINBURGH EHI $1 \mathrm{HZ}$ 